Título artículo / Títol article:

A simple model of herd behavior, a comment

Autores / Autors

Andrea Morone

Revista:

Economics Letters

Versión / Versió:

Preprint

Cita bibliográfica / Cita bibliogràfica (ISO 690):

MORONE, Andrea. A simple model of herd behavior, a comment. Economics Letters, 2012 , vol. 114, no 2.

url Repositori UJI:

http://hdl.handle.net/10234/68660 


\title{
SIMPLE MODEL OF HERD BEHAVIOUR, A COMMENT
}

\author{
Andrea Morone \\ University of Bari \\ a.morone@gmail.com
}

\begin{abstract}
In his 'Simple model of herd behaviour', Banerjee (1992) shows that - in a sequential game - if the first two players have chosen the same action, all subsequent players will ignore their own information and start a herd, an irreversible one. The points of strength of Banerjee's model are its simplicity and the robustness of its results. Its weakness is that it is based on three tie-breaking assumptions, which according to Banerjee minimise herding probabilities. In this paper we analyse the role played by the tie-breaking assumptions in reaching the equilibrium. Even if the overall probability of herding does not change dramatically, the results obtained, which differ from Banerjee's are the following: players' strategies are parameter dependent; an incorrect herd could be reversed; a correct herd is irreversible. There are, in addition, some several cases where available information allows players to find out which action is correct, and so an irreversible correct herd starts.
\end{abstract}

Keywords: Herd behaviour.

JEL classification: D8 


\section{Introduction}

Banerjee in his analysis tries to demonstrate the hypothesis of 'herd behaviour': everyone is doing what everyone else is doing, even when his or her private information suggests acting differently. The model is a sequential decision-making game with one wining action and three possible information states of the Subjects: correct, wrong or no information. Banerjee shows that as soon as the first two players choose the same action, all subsequent players will follow them, independently from their private information. Such an irreversible queue ("lock-in") - caused by the individual rational behaviour to gain a better information position by looking at what other Subjects are doing - may result in a non-optimal equilibrium since the imitated action may be a non-wining one. In other words, considering the society as a whole, it might be more efficient if the individuals could not observe the actions of other individuals, i.e. have less information when taking their decisions.

Banerjee's model has two important features: it has both a very simple and intuitive structure and it gives very strong results. Besides the rationality assumption, we suppose that the decisionmaking process of each player is based on a set of three tie-breaking rules, indicating what he/she has to do in each occasion (e.g. when he/she is indifferent between two or more actions). In other words, "each of these assumptions is made to minimize the possibility of herding" (Banerjee, 1992: 803). It can easily be seen that the rules chosen by Banerjee are not the only plausible ones. In what follows, we will therefore examine if there is any critical relation between the tie-breaking rules and the overall dynamics of the model. In particular, we will show that by choosing an alternative decision rule in case that the first player has no signal, the possibility of an irreversible queue starting from the first player is generally smaller, and consequently also the probability of an inefficient lock-in. 
The main goal of this paper is to present some possible extensions to Banerjee's model, which will show the robustness of the original results, as well as the presence of analytical complexities in the extended models.

\section{2. "A simple model of herd behaviour" under different assumption sets}

Let $A=[0,1] \in R$ be the set of all possible investments, where only $a^{*} \in A$ pays a positive pay-off. Let $S=[0,1] \in R$ be the set of all possible signals, where only $s^{*} \in S$ signals to invests in $a^{*}$. The aim of the game is to invest in $a^{*}$. The pay-off is one when action $a^{*}$ is chosen and zero otherwise. There is a population of $N$ players who take their decision sequentially and in a fixed order. Each player knows the choices made by those before him/her but is not aware of the information on which these choices were based on. Furthermore, let $\alpha$ be the probability of receiving a signal and $\beta$ the probability that the signal is correct.

\subsection{The original tie-breaking rules}

The decision-making process is based on the following three tie-breaking rules (we will fallow the notation adopted by Banerjee and refer to these rules as assumptions):

Assumption A. Whenever a decision-maker has no signal and everyone else has chosen zero, he/she always chooses zero.

Assumption B. When decision-makers are indifferent between following their own signal or someone else's choice, they always follow their own signal.

Assumption C. When a decision-maker is indifferent between two or more of the choices made by the previous decision-makers, he/she chooses to follow the one who has the highest value.

Under these assumptions Benarjee demonstrate that once two subjects choose the same action all subsequent subjects will be in a herd irrespectively of their signals and of the game parameterizations. 


\subsection{New tie-breaking rules}

Replacing Assumption A with the following Assumption A1 it is possible to show that the dynamics of the model changes.

Assumption A1. Whenever the first decision-maker has no signal, he/she chooses randomly an action from the set of all possible actions.

The expected pay-off for the subject 1 is zero under both assumptions A1 and A. Under Assumption A, however, an action equal to zero is a clear signal for the subsequent players that the decision-maker did not receive a signal (since the probability of getting a signal equal to zero is zero). Thus, the first player in the sequence who chooses an action different from zero must have received a signal. In the case that the subsequent player does not receive a signal he/she will follow the choice of his/her predecessor. Since two identical actions are more likely to be correct than incorrect, the next player will follow the queue even if he/she receives a signal suggesting acting

differently, and consequently, all the next players will follow this action. Under Assumption A1 however, the first player will choose an action different from zero in case he/she has not received a signal. Thus, a queue starting from the first player is less informative for the next decision-makers than under Assumption A.

It is possible to show that under this new set of tie-breaking rules $(\mathrm{A} 1, \mathrm{~B}$, and $\mathrm{C})$ it is not always the case that, if all previous subjects choose the same action subsequent subjects will follow them, independently from their private information. To complete our task the following proposition is needed:

Proposition 1. If player $n$ gets a signal and he/she can observe a queue of length $n$ - 1 , following the queue would not always be considered the best strategy.

In this context, two possible cases need to be analysed.

Case 1: player $n-1$ gets a signal and his/her best strategy is following the queue. 
Case 2: the best strategy of player $n-1$ is following his/her signal.

Under Case 1, Proposition 1 is trivial. It is evident that the best strategy of player $n$ is to follow the queue.

Under Case 2, in order to prove Proposition 1 we need some lemmas:

Lemma 1. Let us indicate with $\Psi_{n}$ the event "player $n$ gets the correct signal and observes an incorrect queue of length $n-1$ '. Then

$$
\operatorname{Pr}\left(\Psi_{n}\right)=\alpha \beta\left(\alpha(1-\beta)(1-\alpha)^{n-2}+(1-\alpha)^{n-1}\right)
$$

where $\alpha \beta$ is the probability that player $n$ gets the correct signal, and $(1-\alpha)^{n-1}+\alpha(1-\beta)(1-\alpha)^{n-2}$ is the probability of observing a queue of length $n-1$.

Proof of lemma 1. The only two cases in which such a queue can be observed are: 'player 1 gets an incorrect signal (probability $\alpha(1-\beta)$ ) while all the other players get no signal (probability $\left.(1-\alpha)^{n-2}\right)$; ' all $n-1$ players get no signal (probability $\left.(1-\alpha)^{n-1}\right)$.

Q.E.D.

Lemma 2. Let $\Phi_{n}$ be the event 'player $n$ gets an incorrect signal and observes a correct queue of length $n-1$ '. Then

$$
\begin{aligned}
& \alpha(1-\beta)\left(\alpha \beta \left((\alpha \beta)^{n-2}+\left(\begin{array}{c}
n-2 \\
1
\end{array}\right)(\alpha \beta)^{n-3}(1-\alpha)+\left(\begin{array}{c}
n-2 \\
2
\end{array}\right)(\alpha \beta)^{n-4}(1-\alpha)^{2}+\ldots\right.\right. \\
& \left.\left.\ldots+\left(\begin{array}{c}
n-2 \\
n-3
\end{array}\right)(\alpha \beta)(1-\alpha)^{n-3}+\left(\begin{array}{l}
n-2 \\
n-2
\end{array}\right)(1-\alpha)^{n-2}\right)\right)
\end{aligned}
$$

where $\alpha(1-\beta)$ is the probability that player $n$ gets an incorrect signal; $\alpha \beta$ is the probability that player 1 gets the correct signal; $(\alpha \beta)^{n-2}$ is the probability that all players from 2 to $n$-1 get the correct signal; $\left(\begin{array}{c}n-2 \\ 1\end{array}\right)(\alpha \beta)^{n-3}(1-\alpha)$ is the probability that all players from 2 to $n-1$ get the correct signal and one of them gets no signal. The proof of this Lemma is similar to the previous one. 
Noting that equation (2) is the Newton expansion of a binomial in the power $n$ - 2 , it is possible to rearrange the equation as:

$$
\operatorname{Pr}\left(\Phi_{n}\right)=\left\lfloor\alpha \beta(1+\alpha \beta-\alpha)^{n-2}\right\rfloor
$$

Lemma 3. If player $n$ 's best strategy is to follow his/her signal, than it must also be the case for player $n$ - $k$, where $n, k \in N^{+}$and $n \geq k$

Proof of lemma 3. Let player $n$ 's best strategy be following his/her signal. In other terms: $\operatorname{Pr}\left(\Psi_{n}\right)-\operatorname{Pr}\left(\Phi_{n}\right)>0$. So we have:

$$
\left.\alpha \beta\left(\alpha(1-\beta)(1-\alpha)^{n-2}+(1-\alpha)^{n-1}\right)-\alpha(1-\beta) \operatorname{Pr}\left(\Phi_{n}\right) \alpha \beta(1+\alpha \beta-\alpha)^{n-2}\right\rfloor 0
$$

that is equivalent to

$$
\alpha \beta(1-\alpha \beta)(1-\alpha)^{n-2}-\alpha^{2} \beta(1-\beta)(1+\alpha \beta-\alpha)^{n-2}>0 .
$$

We can rewrite equation (5) as:

$$
\begin{aligned}
& \alpha \beta(1-\alpha \beta)(1-\alpha)^{n-3}(1-\alpha)-\alpha^{2} \beta(1-\beta)(1+\alpha \beta-\alpha)^{n-3}(1+\alpha \beta-\alpha)>0 ; \\
& \alpha \beta(1-\alpha \beta)(1-\alpha)^{n-3}>-\alpha^{2} \beta(1-\beta)(1+\alpha \beta-\alpha)^{n-3} \frac{(1+\alpha \beta-\alpha)}{(1-\alpha)} .
\end{aligned}
$$

Noting that $\frac{(1+\alpha \beta-\alpha)}{(1-\alpha)}>0$ for all $\alpha, \beta \in[0,1]$, it is also true that:

$\alpha \beta(1-\alpha \beta)(1-\alpha)^{n-3}>\alpha^{2} \beta(1-\beta)(1+\alpha \beta-\alpha)^{n-3}$.

We can rewrite the equation (8) as:

$$
\operatorname{Pr}\left(\Psi_{n-1}\right)-\operatorname{Pr}\left(\Phi_{n-1}\right)=\alpha \beta(1-\alpha \beta)(1-\alpha)^{n-3}-\alpha^{2} \beta(1-\beta)(1+\alpha \beta-\alpha)^{n-3}>0
$$

that implies that the best strategy of player $n-1$ is following his/her signal.

Q.E.D.

After having defined and proofed the three lemmas above, we can return to Proposition 1. At this point, in order to prove Proposition 1, we have to show the existence of $\alpha$ and $\beta \in[0,1] \ni^{\prime}$ one of the following conditions is met: 
1. $\operatorname{Pr}\left(\Psi_{n}\right)<\operatorname{Pr}\left(\Phi_{n}\right)$;

2. $\operatorname{Pr}\left(\Psi_{n}\right)>\operatorname{Pr}\left(\Phi_{n}\right)$;

3. $\operatorname{Pr}\left(\Psi_{n}\right)=\operatorname{Pr}\left(\Phi_{n}\right)$, i.e. subjects are indifferent between following the queue or their private information.

Proof of Proposition 1. Let us indicate that $\Im(\alpha, \beta)=\operatorname{Pr}\left(\Phi_{n}\right)-\operatorname{Pr}\left(\Psi_{n}\right)$. Therefore:

$\left.\Im(\alpha, \beta)=\alpha(1-\beta) \alpha \beta(1+\alpha \beta-\alpha)^{n-2}\right\rfloor \alpha \beta\left(\alpha(1-\beta)(1-\alpha)^{n-2}+(1-\alpha)^{n-1}\right)$.

Without loss of generality, let us assume that $\alpha=\beta=x$. Equation (10) can then be rewritten as:

$$
\Im(x)=x^{2}\left(1+x^{2}-x\right)^{2-2}-(1-x)^{n-2}(1+x)
$$

Considering that $\lim _{x \rightarrow 0} \Im(x)=-1$ and $\lim _{x \rightarrow 1} \Im(x)=1$, then for $x=0 \quad$ (respectively $x=1$ ) $\operatorname{Pr}\left(\Psi_{n}\right)>\operatorname{Pr}\left(\Phi_{n}\right)\left(\right.$ respectively $\operatorname{Pr}\left(\Psi_{n}\right)<\operatorname{Pr}\left(\Phi_{n}\right)$ ).

Moreover, the intermediate value theorem guarantees that a value of $x$ $\ni^{\prime} \operatorname{Pr}\left(\Psi_{n}\right)=\operatorname{Pr}\left(\Phi_{n}\right)$ exists.

Q.E.D.

The first result of our paper, thus, is that the equilibrium decision rule is parameter dependent: even if the equilibrium is characterised by extensive herd behaviour, when a queue starts, subjects will not always find themselves locked in. In other words, breaking herd behaviour is possible.

\section{Conclusion}

In this work we extended Banerjee's model of herd behaviour replacing one of its fundamental and 'innocuous' assumptions. More precisely, we replaced Assumption A (whenever a decision-maker has no signal and everyone else has chosen zero, he/she will always choose zero), with Assumption 
A1 (whenever a decision-maker has no signal and everyone else has chosen zero, he/she will always choose randomly among all possible actions).

The consequence of this slight change in the assumptions' set leads to an important alteration in the players' strategy. On the one hand, in Banerjee's model two identical actions are enough to generate an irreversible queue. This fact results in a loss of information with the consequence of a non optimal aggregated result. On the other hand, in the modified model players' strategies are parameter dependent: private information will not be systematically ignored in the presence of a queue.

\section{References}

Banerjee, A. (1992). A Simple Model of Herd Behaviour. Quarterly Journal of Economics, CVII:797-817. 